inappropriate treatment plasma potassium estimations in patients with acute myeloid leukaemia should be made on plasma separated immediately after venepuncture.

We thank Dr M Qureshi for permission to study his patient and Professor M D Rawlins for his help and advice.

1 Danowski TS. The transfer of potassium across the human blood cell membrane. F Biol Chem 1941 ;139:692-705.

2 Pickering TG, Catovsky D. Hypokalaemia and raised lysozyme levels in acute myeloid leukaemia. $Q \mathcal{F}$ Med 1973;42:677-82.

3 Mir MA, Brabin B, Tang OT, Leyland MJ, Delamore IW. Hypokalaemia in acute myeloid leukaemia. Ann Interm Med $1975 ; 82: 54-7$.

4 Tattersall MH, Battersby G, Spiers AS. Antibiotics and hypokalaemia. Lancet $1972 ; \mathrm{i}: 630-1$

5 Block JB, Bonting SL. Sodium-potassium activated adenosine triphosphatase and cation transport in normal and leukaemic leukocytes. Enxymologia Biologica et Clinica 1964;4:183-98.

(Accepted 6 fanuary 1981)

Medical Unit 2 and Department of Laboratory Medicine, Freeman Hospital, Newcastle upon Tyne

P C ADAMS, MRCP, registrar in medicine (present appointment: registrar in general medicine, Royal Victoria Infirmary, Newcastle upon Tyne) $\mathrm{KW}$ WOODHOUSE, MRCP, research associate in clinical pharmacology $M$ ADELA, MD, senior registrar in haematology

A PARNHAM, MSC, biochemist

\section{Brain abscess due to Arachnia propionica}

Arachnia propionica, a member of the Actinomycetaceae, has rarely been described as an agent of human infections. We report the isolation of this organism from a brain abscess and emphasise the problems associated with its identification.

\section{CASE REPORT}

A 32-year-old man presented with a two-day history of severe central headache not relieved by the usual analgesics, falling to the left when walking, and spatial visual difficulties on looking to the left. His medical history indicated that he had developed Eisenmenger's syndrome as a result of an inoperable ventricular septal defect, confirmed by cardiac catheterisation in 1952. During the procedure a left cerebrovascular accident had occurred, which had resulted in continuing convulsions, right-sided hemiparesis, and growth retardation. He had been started on prophylactic phenoxymethylpenicillin $250 \mathrm{mg}$ twice daily.

Four months before presentation he had been admitted to hospital for treatment and control of cardiac failure. Shortly after discharge he stopped the penicillin prophylaxis. On readmission he was subjected to a full examination and special investigations. A computed tomography scan showed generalised atrophy of the left cerebral hemisphere and a lesion in the right occipital lobe compatible with an abscess. No primary focus of infection was found. He was started on intravenous ampicillin $2 \mathrm{~g}$ four times daily and gentamicin $80 \mathrm{mg}$ and metronidazole $500 \mathrm{mg}$ thrice daily and underwent craniotomy, when an abscess close to the medial surface and above the calcarine sulcus of the right occipital lobe was drained and the wall excised.

Pus was cultured aerobically on blood agar, CLED agar, and heated blood agar, and anaerobically on blood agar and a supplemented lysed blood agar containing gentamicin $5 \mathrm{mg} / 1$, cysteine hydrochloride $0.05 \%$, yeast extract $0.5 \%$, and vitamin $\mathrm{K}_{1} 0.0002 \%$. Microscopy showed numerous leucocytes, Gram-positive cocci, and branching Gram-positive bacilli. After 48 hours' incubation results of aerobic culture were negative while anaerobic culture produced an anaerobic Gram-positive coccus subsequently identified as Peptostreptococcus micros and a branching anaerobic Grampositive bacillus identified as $A$ proprionica. Identification of isolates was based on morphological studies, routine biochemical tests, and analysis of the end products of glucose fermentation as described by Holdeman et al. ${ }^{3}$ He was treated with intravenous penicillin and metronidazole and discharged three weeks later taking oral penicillin.

\section{Comment}

$A$ propionica isolations have usually been associated with lesions of the cervical lymphocutaneous tissues, lacrimal glands, lung, abdomen, ${ }^{2}$ and bone. ${ }^{3}$ We have found no reports of a brain abscess due to this organism. $A$ propionica is a resident of the oropharynx, as is $P$ micros, and the organisms isolated from the abscess probably originated from this site despite the absence of oral disease.

Identification of $A$ propionica in the microbiology laboratory can be difficult. Morphologically and biochemically the organism is similar to Actinomyces israelii and clinically may produce a disease that is indistinguishable. Three tests are available to differentiate the two organisms-namely, the demonstration of diaminopimelic acid in the cell wall of $A$ propionica, specific fluorescence antibody staining, and the demonstration of the production of propionic acid by gas-liquid chromatography from glucose fermentation by $A$ propionica. ${ }^{2}$ As the first two tests are beyond the scope of most clinical laboratories, the importance of carrying out gas-liquid chromatography analysis of a glucose broth for all organisms resembling Actinomyces cannot be overemphasised.

Both organisms isolated from the abscess were sensitive to penicillin. Hence an important question is raised regarding the use of prophylactic antibiotics, in this case phenoxymethylpenicillin, in patients with cardiac anomalies, either congenital or acquired. Should the patient take subtherapeutic dosages of prophylactic antibiotics for long periods and risk acquiring resistant oral microflora or stop prophylaxis and risk infection with commensal micro-organisms?

In our patient oral prophylaxis with phenoxymethylpenicillin was justified as we assume that a bacteraemic episode had occurred a few weeks before presentation while he was not taking antibiotics. Although it is recommended that prophylaxis is indicated only when a patient is subjected to some manipulative procedure known to cause bacteraemia, ${ }^{4}$ phenoxymethylpenicillin had been continued in our patient, albeit at subtherapeutic dosages, after he was discharged, in an attempt to prevent a further bacteraemic episode.

We thank Dr M Lee for permission to report this case, and Dr K T Karthigasu for helpful discussion in preparing the manuscript.

${ }^{1}$ Holdeman LV, Cato EP, Moore WEC, eds. Anaerobe laboratory manual. 4th ed. Blacksburg: Virginia Polytechnic Institute and State University, 1977.

2 Brock DW, Georg LK, Brown JM, Hicklin MD. Actinomycosis caused by Arachnia propionica. Am f Clin Pathol 1973;59:66-77.

${ }^{3}$ Conrad SE, Breivis J, Fried MA. Vertebral osteomyelitis, caused by Arachnia propionica and resembling actinomycosis. $\mathcal{F}$ Bone foint Surg ( $A m)$ 1978;60-A :549-53.

${ }^{4}$ Petersdorf RG. Antimicrobial prophylaxis of bacterial endocarditis. Am $\mathcal{F}$ Med 1978;65:220-23.

(Accepted 6 fanuary 1981)

Department of Clinical Microbiology, University of Western Australia, Queen Elizabeth II Medical Centre, Shenton Park, Western Australia 6008

T V RILEY, BAPPSC, MASM, technologist

A K OTT, MB, CHB, registrar

\section{Legionnaires' disease and abscess of appendix}

While gastrointestinal symptoms, particularly diarrhoea and abdominal distension, are common in legionnaires' disease, ${ }^{1}$ a surgical "acute abdomen" has not previously been reported in this condition. I report on a patient with legionnaires' disease complicated by an abscess of the appendix.

\section{Case report}

A 48-year-old engineer was admitted to this hospital with a seven-day history of headache, rigors, and fever. Three days before admission he had developed an unproductive cough without chest pain or dyspnoea; treatment with co-trimoxazole had been started. The next day he had developed central abdominal pain, which had worsened and localised in the right iliac fossa. He had been anorexic and was constipated. He had become ill on the day he returned from a 10 -day holiday in Benidorm.

On examination he was seen to be feverish and toxic. He was tachypnoeic with clinical signs of a left basal pneumonia. There was a tender, $3-\mathrm{cm}$ diameter mass palpable in the right iliac fossa, with guarding and rebound tenderness.

Haemoglobin concentration was $15.9 \mathrm{~g} / \mathrm{dl}$ and white cell count $10 \times 10^{\circ} / 1$ 
$\left(10000 / \mathrm{mm}^{3}\right)$ with $78 \%$ neutrophils. Plasma sodium concentration was $121 \mathrm{mmol}(\mathrm{mEq}) / \mathrm{l}$, and urea and potassium concentrations were normal. Serum aspartate aminotransferase activity was $180 \mathrm{IU} / 1$ (normal 3-35 IU/1) and serum amylase activity $390 \mathrm{IU} / 1$ (normal $40-160 \mathrm{IU} / 1$ ). A chest $x$-ray film showed extensive consolidation in the left lung. No pathogenic organisms were isolated from sputum or blood cultures. Indirect fluorescence tests on admission and days 8,11 , and 21 showed antibody titres to Legionella pneumophila of $1 / 8,1 / 32,1 / 64$, and $1 / 128$ respectively. There was no serological evidence of infection with influenza, respiratory syncytial virus or adenovirus, group B chlamydia, or Mycoplasma pneumoniae.

As legionnaires' disease was suspected on admission he was given erythromycin $500 \mathrm{mg}$ four times daily for 10 days. The abscess of the appendix was treated conservatively with intravenous fluids, and gentamicin and metronidazole were given for 10 days. On this regimen his condition improved steadily, the pneumonia having cleared clinically and radiologically after 12 days in hospital. Appendicectomy was performed two months later. Histological examination showed a subacute abscess with no specific features. He has remained asymptomatic since the operation.

\section{Comment}

Although abdominal symptoms are often present in patients with legionnaires' disease, structural lesions of the gastrointestinal tract have not been found at necropsy. The symptoms of appendicitis were this patient's main complaint-in fact, despite extensive pneumonia, he complained of a dry cough only on direct questioning. While this may have been a chance association, I believe that this complication might be overlooked in a patient severely ill with respiratory failure or encephalopathy, and if not treated correctly might have a deleterious effect on the outcome.

I thank Dr T Rowbotham for the legionella serology, Mr T Brennan for his surgical advice, and Dr J Stevenson for permission to report on this patient.

1 Anonymous. Legionnaires' disease. $B r$ Med f 1978;ii:1319-20.

Department of Infectious Diseases, Seacroft Hospital, Leeds LS14 6UH

PAUL HOLT, MRCP, senior registrar

\section{Spacing between doses on a thrice-daily regimen}

The effect of drug treatment is related not only to the number of doses taken but also to the timing of doses. This is particularly true for drugs with a narrow therapeutic range and a short elimination half-life. While considerable variations in the patients' interpretations of the prescription label have been reported, ${ }^{1}$ the spacing between doses has not been studied objectively. We used a recording medication monitor ${ }^{2}$ to study the spacing between doses by patients on a thrice-daily regimen and to study the validity of interview data on the timing of doses.

\section{Patients, methods, and results}

The medication behaviour of 82 patients ( 45 men, 37 women) aged 56-90 years who were being treated with pilocarpine thrice daily for simple glau coma was studied for three weeks with a medication monitor, which recorded the date and hour each time the medication bottle was opened. ${ }^{2}$ Other sources of the drug were controlled, and the patients were not told that their drug-taking was being monitored until all the data had been collected.
From each monitor record we determined the three times (more than two hours apart) when doses were most frequently taken. For each patient the spacing between doses was then determined as the intervals between these times. Monitor records from five patients were lost for technical reasons. The mean of the first, second, and third intervals was $6.4,6.5$, and $11 \cdot 1$ hours respectively (table). The third interval was 12 hours or more for 30 $(39 \%)$ patients. Only four patients took the drug at eight-hourly intervals. In the most extreme case the patient took the drug at intervals of three three, and 18 hours. The patients studied took their drug at relatively similar intervals from day to day: $68(88 \%)$ took at least $80 \%$ of their doses within one hour of their usual time.

After the recording period the patients were asked at which hours of the day they usually took the drug. Five patients were lost from these interviews. Comparisons with data from the monitor showed good agreement: for $85 \%$ of the intervals the difference was one hour or less. Only nine patients reported an interval with a deviation of more than two hours from that recorded by the monitor.

\section{Comment}

Although interview data on missed doses are often inaccurate, ${ }^{3}$ our findings show that interviews with patients were accurate in determining the spacing between doses. In studies of medication compliance data on the spacing between doses gained at interview may be combined with other methods to determine the number of doses takenfor example, pill count. In routine medical practice interviews may be useful for obtaining information about irregular dosage intervals and as a first step towards improving the situation.

A striking finding was the irregular timing of doses among patients on a thrice-daily regimen. Assuming that the effective duration of the drug is about eight hours, ${ }^{4}$ it is probable that long night intervals unfavourably influence the result of the treatment. Among patients with a twice-daily regimen Hermann found a tendency to spread doses more regularly, with an average night interval of 13.6 hours. There is evidence that the proportion of missed doses is lower with a twice-daily than with a thrice-daily regimen. ${ }^{5}$

Hence, both in terms of missed doses and of spacing between doses, drugs prescribed twice daily will probably be more properly administered than those prescribed three times a day. When it is not possible to reduce the frequency of doses tailoring of doses to daily routines may improve compliance both in terms of spacing and of missed doses. 5

This study was supported in part by grants from the Swedish Medica Research Council.

Requests for reprints should be sent to Lars Alfredsson, Department of Social Medicine, Huddinge University Hospital, S-141 86 Huddinge, Sweden.

${ }^{1}$ Hermann F. The outpatient prescription label as a source of medication errors. Am $\mathcal{F}$ Hosp Pharm 1973;30:155-9.

2 Norell SE, Granström P-A, Wassén R. A medication monitor and fluorescein technique designed to study medication behaviour. Acta Ophthal mol (Copenh) 1980;58:459-67.

${ }^{3}$ Norell SE. Accuracy of patient interview and estimates by clinical staff in determining medication compliance. Soc Sci Med (in press).

4 Heilman K, Richardson KT, eds. Glaucoma: conceptions of a disease. Stuttgart: G Thieme, 1978: 272.

5 Norell SE. Medication behaviour: a study of outpatients treated with pilocarpine eye drops for primary open-angle glaucoma. Acta Ophthalmol [Suppl] (Copenh) 1980;143.

(Accepted 9 Fanuary 1981)

Department of Social Medicine, Karolinska Institutet, Huddinge University Hospital, Huddinge, Sweden

LARS S ALFREDSSON, BA, research assistant

STAFFAN E NORELL, MD, physician

Intervals between doses of pilocarpine prescribed three times daily

\begin{tabular}{|c|c|c|c|c|c|c|c|c|c|c|c|c|c|c|c|c|c|c|c|c|}
\hline Duration of interval (hours) & 0 & $-1 \frac{1}{2}$ & $-2 \frac{1}{2}$ & $-3 \frac{1}{2}$ & $-4 \frac{1}{2}$ & $-5 \frac{1}{2}$ & $-6 \frac{1}{2}$ & $-7 \frac{1}{2}$ & $-8 \frac{1}{2}$ & $-9 \frac{1}{2}$ & $-10 \frac{1}{2}$ & $-11 \frac{1}{2}$ & $-12 \frac{1}{2}$ & $-13 \frac{1}{2}$ & $-14 \frac{1}{2}$ & $-15 \frac{1}{2}$ & $-16_{2}^{\frac{1}{2}}$ & $-17 \frac{1}{2}$ & $-18 \frac{1}{2}$ & -20 \\
\hline \multicolumn{21}{|c|}{ First interval } \\
\hline No of patients & & & & 5 & 7 & 11 & 15 & 17 & 16 & 5 & 1 & & & & & & & & & \\
\hline \multicolumn{21}{|c|}{ Second interval } \\
\hline \multicolumn{21}{|c|}{$\begin{array}{cc}\text { Third interval } \\
1\end{array}$} \\
\hline
\end{tabular}

